\title{
Dielectric Relaxation Phenomena in SBN Single Crystals Doped with Ce
}

\author{
Yu. Korchak ${ }^{a}$, V. KapustianyK ${ }^{b}$, B. Fedor ${ }^{c}$, I. GirnyK ${ }^{b}$ And Yu. Eliyashevskyi ${ }^{b}$ \\ ${ }^{a}$ Ivan Franko National University of Lviv, 107 Tarnavskyi Str., L'viv, 79017, Ukraine
}

${ }^{b}$ Scientific-Technical and Educational Centre of Low-Temperature Studies, Ivan Franko National University of Lviv 50 Dragomanov Str., Lviv, 79005, Ukraine

${ }^{c}$ Hetman P. Sahaydachnyi Academy of the Army, 32 Gvardiyska Str., Lviv, 79012, Ukraine

(Received September 24, 2010; revised version November 25, 2010; in final form February 25, 2011)

\begin{abstract}
The dielectric study of $\mathrm{Sr}_{0.73} \mathrm{Ba}_{0.27} \mathrm{Nb}_{2} \mathrm{O}_{6}$ :Ce (SBN) crystals along [010] crystallographic axis was performed in the temperature region of $310-360 \mathrm{~K}$ and frequency range from $25 \mathrm{~Hz}$ up to $1 \mathrm{MHz}$. The thermal dipole relaxation of quasi-Debye-type for this orientation of sample was observed in both investigated structural phases. The phase transition was most clearly seen from the temperature dependence of the relaxation time at $T_{\mathrm{c}}=320 \mathrm{~K}$. The relaxation processes were related to the collective oscillations of the $\mathrm{Nb}-\mathrm{O}$ bonds in the two different type corner-sharing $\mathrm{NbO}_{6}$ octahedra aligned along $c$-axis.
\end{abstract}

PACS: 77.22.-d, 77.22.Ch, 77.22.Gm, 77.80.Jk

\section{Introduction}

The tungsten bronze type relaxor ferroelectric material - strontium barium niobate $\mathrm{Sr}_{1-x} \mathrm{Ba}_{x} \mathrm{Nb}_{2} \mathrm{O}_{6}(\mathrm{SBN})-$ is considered as a perspective material for applications in the areas of pyroelectricity, piezoelectricity, electrooptics, photorefractive optics and non-linear optics [1,2].

A first structural characterization of this type of materials has been made in 1968 [3] for the composition $x=0.75$. The ferroelectric relaxor SBN belongs to the structure type of tetragonal tungsten bronze (TTB) with the space group $P 4 b m$ at room temperature (see Fig. 1) [4]. The unit cell contains ten corner-sharing $\mathrm{NbO}_{6}$ octahedra of two types $\left(\left[\mathrm{Nb}(1) \mathrm{O}_{6}\right]\right.$ and $\left.\left[\mathrm{Nb}(2) \mathrm{O}_{6}\right]\right)$, building the chains along polar axis. $\mathrm{Sr}$ and $\mathrm{Ba}$ occupy the tetragonal and pentagonal positions; while the smaller 12-fold coordinated tetragonal site is occupied only by Sr. Both Sr and Ba were found in the larger 15-fold coordinated pentagonal site. The trigonal positions are vacant. This cation distribution was recently confirmed for SBN crystals with $x=0.61$ [5] and 0.75 [6]. The compositional range of the ferroelectric TTB phase usually was defined within the values $x=0.25-0.75$.

The physical properties of such compounds possess the following particularities. The strontium barium niobate displays the relaxor transition at the point relatively close to the room temperature. It changed from $503 \mathrm{~K}$ for $x=0.32$ to $283 \mathrm{~K}$ for $x=0.82$ [7-9]. The anomalies of different physical characteristics, connected with this transition of SBN from non-polar (point group $D_{4 h}[10]$ )

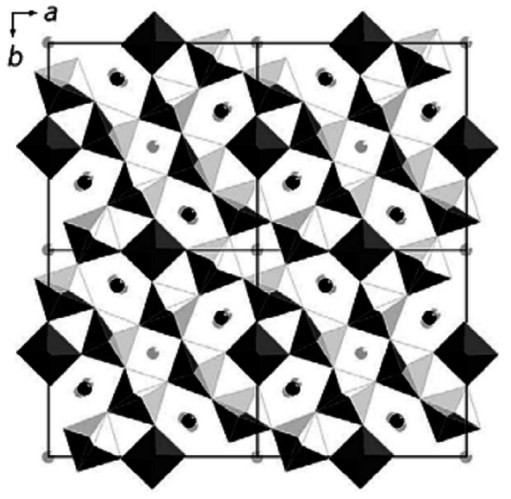

Fig. 1. c-cut of SBN crystal structure [4].

into the polar phase $\left(C_{4 v}\right.$ [11]), usually are smeared. For example, the dielectric permittivity measured in a weak electric field manifests a weakly denominated broad maximum at a certain temperature $\left(T_{\mathrm{m}}\right)$. Rare-earths (RE) doping lower the phase transition temperature of the crystals and smear the phase transition without any abatement of the optical quality [12]. In the temperature area of the phase transition typical dispersion of the dielectric permittivity and dielectric losses occurs in the low-frequency range. At the same time the narrow "extended" loops of the dielectric hysteresis exist within the large interval both below and above the phase transition temperature slowly degrading at heating [13]. 
The smearing of the phase transition followed by the broad anomalies of different physical characteristics of TTB are caused by the wide variation of the non-equivalent crystallographic positions in its structure [14], leading to appearance of the local distortions of symmetry and internal electric fields in the wide temperature range, covering $T_{\mathrm{m}}$. For SBN crystal such a behavior is connected with disordering of the ion $\mathrm{Sr}$ between the two cation lattice sites. As a result the entire crystal appears as a non-polar matrix, in which small spontaneous polarized areas (nanodomains) are randomly embedded [14].

The aim of this report consists in investigations of the dielectric relaxation in a TTB crystal of SBN for the case when the electrodes are set along [010] axis in contrast to a well-studied dielectric relaxation along [001] polar axis.

\section{Experimental}

The investigated $\mathrm{Sr}_{0.73} \mathrm{Ba}_{0.27} \mathrm{Nb}_{2} \mathrm{O}_{6}$ (SBN73) crystals have been grown using a Czochralski method with $\mathrm{CeO}_{2}$ as a dopant (2 wt\%). The doping with a small concentration of Ce usually is used for studies of the photorefractive effect in this crystal which appears as the most perspective material for dynamic holography.

For the dielectric measurements the crystalline platelets of $a$-cut with the thickness of $1-2 \mathrm{~mm}$ and the area of $25-35 \mathrm{~mm}^{2}$ were used. After polishing the samples were covered by conducting silver-paste electrodes. The dielectric parameters were measured with an accuracy of nearly $1 \%$ at different frequencies of the measuring electric field (from $25 \mathrm{~Hz}$ up to $1 \mathrm{MHz}$ ) using a computerized site based on E7-20 multifrequency LCR-meter. The applied ac electric field did not exceed $1 \mathrm{~V} / \mathrm{cm}$. For the temperature investigations the samples were placed in a liquid nitrogen cryostat. The rate of the temperature change was $0.5 \mathrm{~K} / \mathrm{min}$.

\section{Results and discussion}

The temperature dependences of the real $\varepsilon^{\prime}$ and imaginary $\varepsilon^{\prime \prime}$ parts of the dielectric permittivity measured along [010] axis of SBN73:Ce are presented in Fig. 2. The specific dispersion of these parameters is observed. The detailed investigation of the dielectric relaxation was performed in the region lying above room temperature. It is clearly seen that $\varepsilon_{b}^{\prime}$ decreases with increasing frequency (Fig. 2a). At the same time, we observed decreasing (above $20 \mathrm{kHz}$ ) and broadening of the $\varepsilon_{b}^{\prime \prime}$ maxima with an increasing frequency (Fig. 2b) contrary to the behavior characteristic to a pure relaxor [15]. Besides, as one can see from Fig. 2b the position of the maximum corresponding to the dielectric relaxator is shifted toward higher temperatures at increase of the frequencies of the measuring electric field. It is necessary to note that the dielectric dispersion which was found to be fitted to the well-known Vogel-Fulcher law in the frequency range of $100 \mathrm{~Hz}-1 \mathrm{MHz}$ for the measuring electric field applied along [001] crystallographic axis of SBN compounds [15],

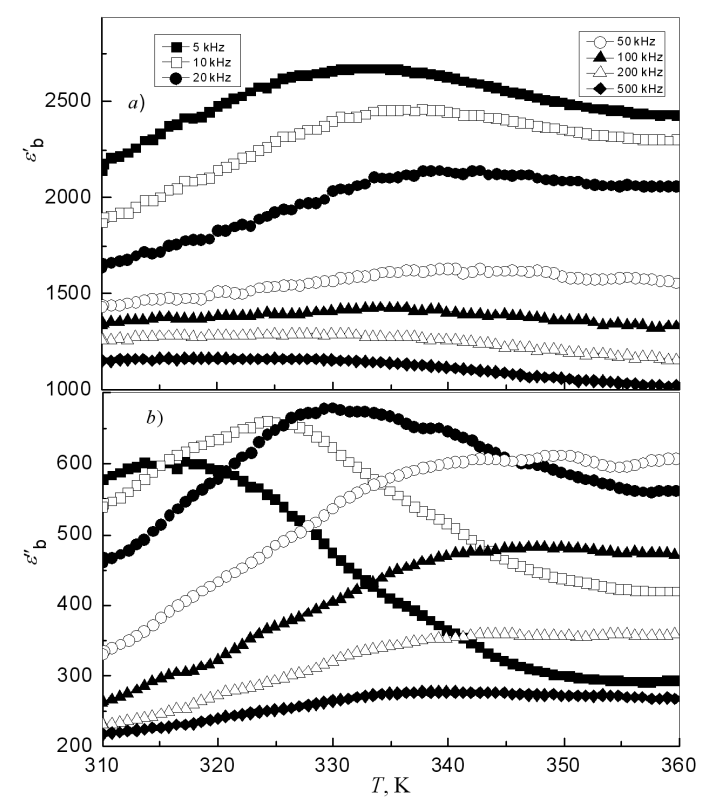

Fig. 2. Temperature dependences of the real (a) and imaginary (b) parts of dielectric permittivity of SBN73:Ce crystal obtained at different frequencies of measuring electric field.

should be considered as a characteristic feature of the relaxor compounds.

In our case the dielectric spectra show a quasi-Debye-type behavior in the high frequency part of the dielectric spectra, considerably deviating from the Debye-type behavior at lower frequencies which implies a more complicate procedure of approximation. As it is seen from Fig. 2, the temperature dependences of the dielectric permittivity do not show clear anomalies that would be related to the phase transitions, first of all due to the masking role of the dielectric dispersion.

It is known [16] that in general for an inhomogeneous insulator the spectra of the real and imaginary parts of the dielectric permittivity are described by

$$
\begin{aligned}
& \varepsilon^{\prime}(\omega)=\varepsilon_{\infty}+\frac{\varepsilon_{0}-\varepsilon_{\infty}}{1+\omega^{2} \tau^{2}}, \\
& \varepsilon^{\prime \prime}(\omega)=\left(\varepsilon_{0}-\varepsilon_{\infty}\right) \frac{\omega \tau}{1+\omega^{2} \tau^{2}},
\end{aligned}
$$

where $\omega$ is the frequency of measuring field, $\tau=1 / 2 \pi f_{\mathrm{r}}$ is the average relaxation time, $f_{\mathrm{r}}$ is the loss or imaginary dielectric permittivity peak frequency, $\varepsilon_{0}$ is the permittivity obtained by static measurements, $\varepsilon_{\infty}$ denotes the high-frequency limit. Figure 3 shows that the frequency dependences of $\varepsilon^{\prime}$ and $\varepsilon^{\prime \prime}$ within $1 \mathrm{kHz}-1 \mathrm{MHz}$ measured at $320 \mathrm{~K}$ along [010] crystallographic axis of SBN73:Ce should be connected with relaxation process. The nature of this relaxation process would be clear only when one considers its temperature evolution.

The dispersive equation for $\varepsilon^{*}(\omega)=\varepsilon^{\prime}(\omega)-\mathrm{i} \varepsilon^{\prime \prime}(\omega)$ in the case of the pure Debye-type relaxation may be 


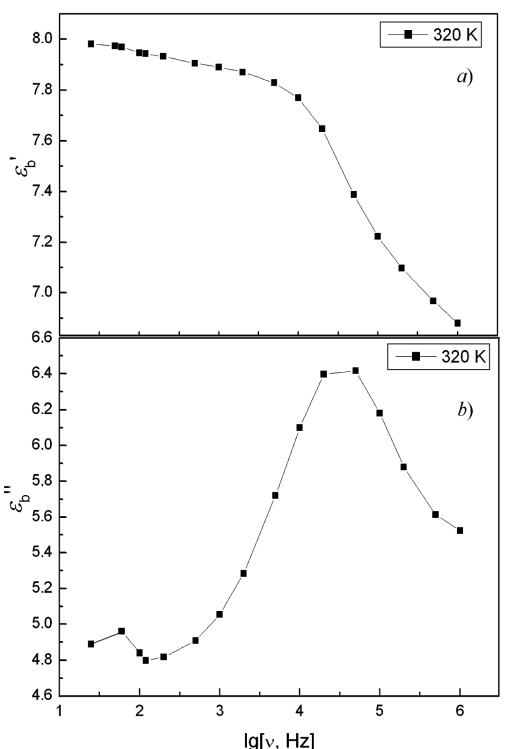

Fig. 3. Frequency dependences of $\varepsilon_{b}^{\prime}$ (a) and $\varepsilon_{b}^{\prime \prime}$ (b) in the semilogarithmic scale at $T=320 \mathrm{~K}$.

obtained from the Debye equation [17]:

$$
\frac{\varepsilon^{*}(\omega)-\varepsilon_{\infty}}{\varepsilon_{0}-\varepsilon_{\infty}}=\frac{1}{1+\mathrm{i} \omega \tau} .
$$

Equations (1) and (2) should be considered as sequences of relation (3). In this case the relaxation time follows a temperature dependence of the Arrhenius type [18]:

$$
\tau=\tau_{0} \exp \left(\frac{E_{\mathrm{r}}}{k T}\right),
$$

where $\tau_{0}$ is the relaxation time at infinite temperature which is inverse to the oscillation frequency of the dipole (or charge) in its potential well and $E_{\mathrm{r}}$ stands for the activation energy of the relaxation.

Examples of the Cole-Cole diagrams $\left(\varepsilon_{b}^{\prime \prime}\right.$ versus $\left.\varepsilon_{b}^{\prime}\right)$ for the SBN73:Ce crystal obtained at different temperatures within the both phases are shown in Fig. 4. Although the shape of the plots is close to the semicircles, they deviate from a pure Debye relaxation behavior since the semicircles are not centered on the $\varepsilon_{b}^{\prime}$-axis. This implies some distribution of relaxation times and means that the observed relaxation is of polydispersive type. In this case the complex dielectric constant can be written as [19]:

$$
\varepsilon^{*}(\omega)=\frac{\varepsilon_{0}-\varepsilon_{\infty}}{1+(\mathrm{i} \omega \tau)^{1-\alpha}}+\varepsilon_{\infty},
$$

where $\alpha$ is an empirical parameter $(0<\alpha<1)$ which measures the degree of deviation from the Debye model. The value of $\alpha$ is not equal to zero when the dipoles (relaxators) interact with each other.

The Cole-Cole plot correlates with the corresponding frequency dependence of the $\varepsilon^{\prime}$ (Fig. 3). We obtain the best fit to (4) with $\alpha=0.295 \pm 0.005$ for $T=315 \mathrm{~K}$ and $\alpha=0.320 \pm 0.005$ for $T=350 \mathrm{~K}$. Analyzing the

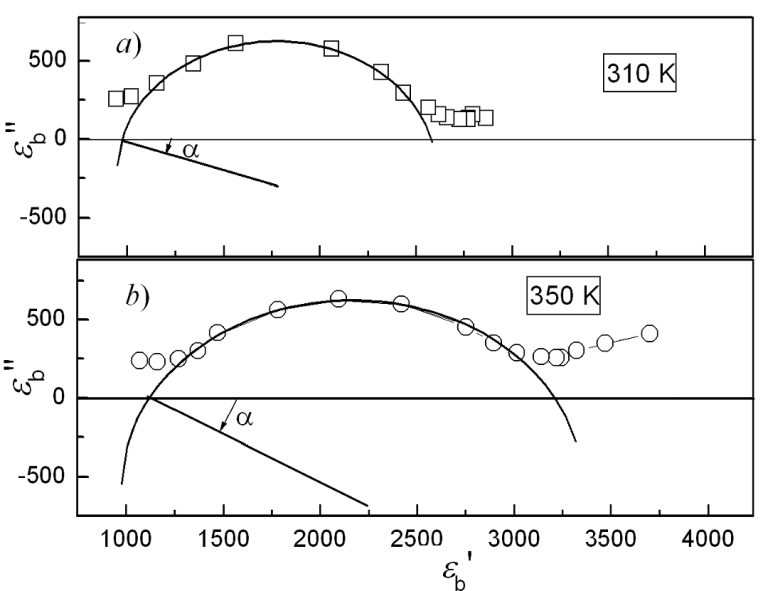

Fig. 4. Cole-Cole diagrams for SBN73:Ce crystal obtained at $210 \mathrm{~K}(\mathrm{a})$ and $350 \mathrm{~K}(\mathrm{~b})$.

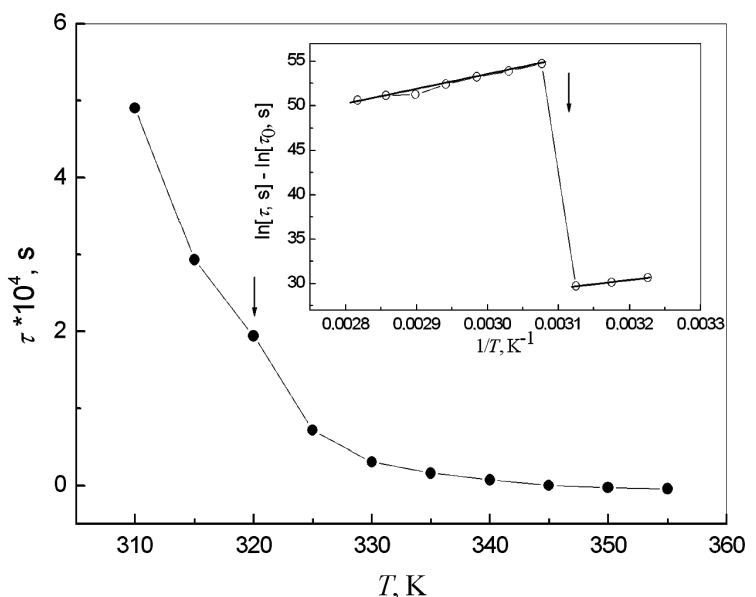

Fig. 5. Relaxation time vs. temperature. Inset presents the Arrhenius plot for the relaxation.

Cole-Cole diagrams for different temperatures using the well-known methods [20] one could calculate the values of the relaxation time. For the observed relaxator these parameters were found to be $10^{-4}-10^{-6} \mathrm{~s}$.

The temperature dependence of the relaxation time with clear anomaly at $T_{\mathrm{c}}=320 \mathrm{~K}$ (phase transition temperature) are shown in Fig. 5. The inverse temperature dependence of $\ln \tau-\ln \tau_{0}$ manifests the jump-like anomaly at the phase transition point $T_{\mathrm{c}}=320 \mathrm{~K}$ (Fig. 5, inset), too. It manifests a linear character in the logarithmic scale within the framework of every investigated phase (Fig. 5, inset). That is why the variation of $\ln \tau$ versus $1 / T$ plot calculated on the basis of measurements performed along [010] could be fitted with the exponential activation law (3). The best fit parameters are $\tau_{0}=2.38 \times 10^{-17} \mathrm{~s}, E_{\mathrm{r}}=0.79 \mathrm{eV}$ for $T<T_{\mathrm{c}}$ and $\tau_{0}=1.25 \times 10^{-28} \mathrm{~s}, E_{\mathrm{r}}=1.41 \mathrm{eV}$ for $T>T_{\mathrm{c}}$.

On the basis of the available structural [21] and dielectric data for related materials [22] one can suppose that 
the determined values of the relaxation time and the activation energy $E_{\mathrm{a}}$ would be related to the collective oscillations of the short strong and long weak $\mathrm{Nb}-\mathrm{O}$ bonds in the two different type corner-sharing $\mathrm{NbO}_{6}$ octahedra aligned along $c$-axis.

\section{Conclusion}

The dielectric dispersion study of $\mathrm{Sr}_{0.73} \mathrm{Ba}_{0.27} \mathrm{Nb}_{2} \mathrm{O}_{6}$ : $\mathrm{Ce}$ crystals crystallographic axis was performed in the temperature region of 310-360 K and frequency range from $25 \mathrm{~Hz}$ up to $1 \mathrm{MHz}$ on the cuts perpendicular to [010] direction.

Thermal dipole relaxation of quasi-Debye-type for this orientation of sample was observed in both investigated structural phases in contrast to a relaxor relaxation behavior along [001] axis characteristic for the same compound. One can suppose that pure ferroelectricity occurs in the direction perpendicular to the crystallographic $c$-axis.

The phase transition was most clearly seen at $T_{\mathrm{c}}=$ $320 \mathrm{~K}$ in the temperature dependence of the relaxation time. Complex approximation of the dielectric spectra allowed to determine the values of the activation energy: $E_{\mathrm{r}}=0.79 \mathrm{eV}$ for $T<T_{\mathrm{c}} ; E_{\mathrm{r}}=1.41 \mathrm{eV}$ for $T>T_{\mathrm{c}}$. The relaxation processes were related to the collective oscillations of the $\mathrm{Nb}-\mathrm{O}$ bonds in the two different type corner-sharing $\mathrm{NbO}_{6}$ octahedra aligned along $c$-axis. This means that the elastic interactions between the octahedra in the $a-b$ plane should give a strong contribution into the ferroelectric properties of SBN.

\section{References}

[1] J.R. Oliver, R.R. Neurgaonkar, L.E. Cross, J. Appl. Phys. 64, 37 (1988).

[2] M. Miyagi, Ya. Sugiyama, Sh. Yagi, I. Hatakeyama, Jpn. J. Appl. Phys. 33, 1417 (1994).

[3] P.B. Jamieson, S.C. Abrahams, J.L. Bernstein, J. Chem. Phys. 48, 5048 (1968).

[4] V.I. Simonov, Nature 11, 4 (2003) (in Russian).
[5] T.S. Chernaya, B.A. Maksimov, I.V. Verin, L.I. Ivleva, V.I. Simonov, Crystallogr. Rep. 42, 375 (1997).

[6] T.S. Chernaya, B.A. Maksimov, T.R. Volk, L.I. Ivleva, V.I. Simonov, Phys. Solid State 42, 1716 (2000).

[7] J. Ravez, A. Simon, J. Solid State Chem. 162, 260 (2001).

[8] C. Elassalde, J. Ravez, J. Mater. Chem. 10, 681 (2000).

[9] C. David, T. Granzow, A. Tunyagi, M. Woehlecke, T. Woike, K. Betzler, M. Ulex, M. Imlau, R. Pankrath, Phys. Status Solidi A 201, R49 (2004).

[10] A.S. Bhalla, R. Guo, L.E. Cross, G. Burus, F.H. Dacol, R.R. Neurgaonkar, Phys. Rev. B 36, 2030 (1987).

[11] M.E. Lines, A.M. Glass, Principles and Applications of Ferroelectrics and Related Materials, Mir, Moscow 1981 (in Russian).

[12] T. Volk, L. Ivleva, P. Lykov, N. Polozkov, V. Salobutin, R. Pankrath, M. Woehlecke, Opt. Mater. 18, 179 (2001).

[13] W.H. Huang, D. Viehland, R.R. Neurgaonkar, J. Appl. Phys. 76, 490 (1994).

[14] L.E. Cross, Ferroelectrics 76, 241 (1987).

[15] I.P. Rayevskiy, V.V. Yeremkin, V.G. Smotrakov, Ye.S. Gagarina, M.A. Malitskaya, Techn. Phys. Lett. 25, 47 (1999) (in Russian).

[16] Yu. Poplavko, Physics of Dielectrics, Vyshcha Shkola, Kyiv 1980 (in Russian).

[17] P. Debye, Polar Molecules, Dover Publ. Inc., New York 1945.

[18] J.C. Badot, N. Baffier, A. Fourier-Lamer, Ph. Colomban, J. Phys. 48, 1325 (1987) (in France).

[19] J.C. Badot, N. Baffier, A. Fourier-Lamer, Ph. Colomban, Solid State Ion. 28-30, 1617 (1988)

[20] A. Chelkowski, Dielectric Physics, Elsevier, Amsterdam 1980.

[21] S. Podlozhenov, H.A. Graetsch, Ju. Schneider, M. Ulex, M. Wöhlecke, K. Betzler, Acta Crystallogr. B 62, 960 (2006).

[22] A. Simon, G.-T. Joo, M. Maglione, J. Ravez, Solid State Sci. 9, 52 (2007) 